\title{
Maternal Stress Affects Fetal Growth but Not Developmental Instability in Rabbits
}

\author{
Jessica Bots ${ }^{1,+}$, Matteo Breno ${ }^{1,+}$, Luc De Schaepdrijver ${ }^{2}$ and Stefan Van Dongen ${ }^{1, *}$ \\ 1 Evolutionary Ecology Group, Department of Biology, University of Antwerp, 2610 Antwerp, Belgium; \\ jessica.bots@uantwerpen.be (J.B.); breno.matteo@gmail.com (M.B.) \\ 2 Drug Safety Sciences, Janssen Research \& Development, 2340 Beerse, Belgium; LDSCHAEP@its.jnj.com \\ * Correspondence: stefan.vandongen@uantwerpen.be; Tel.: +32-3-265-3336 \\ + These authors contributed equally to this work.
}

Academic Editor: John H. Graham

Received: 17 August 2016; Accepted: 18 September 2016; Published: 27 September 2016

\begin{abstract}
Developmental instability (DI), often measured by fluctuating asymmetry (FA) or the frequency of phenodeviants (fPD), is thought to increase with stress. However, specifically for stressors of maternal origin, evidence of such negative associations with DI is scarce. Whereas effects of maternal stress on DI have predominately been examined retroactively in humans, very little is known from experiments with well-defined stress levels in animal model systems. The aim of this study was to examine the effects of maternal exposure to three doses (plus a control) of a toxic compound affecting maternal condition on DI of their offspring in rabbits. Presence of maternal stress induced by the treatment was confirmed by a decrease in food consumption and weight gain of gravid females in the medium and high dose. Major abnormalities and mortality were unaffected by dose, suggesting the lack of toxic effects of the compound on the offspring. In spite of string maternal stress, offspring FA did not increase with dose. The treatment did lead to elevated fPD, but most were transient, reflecting growth retardation. Furthermore, a consistent association between fPD and FA was absent. These findings indicate that DI is not increased by maternal stress in this animal model.
\end{abstract}

Keywords: maternal stress; developmental instability; fluctuating asymmetry; phenodeviants; rabbit; Oryctolagus cuniculus

\section{Introduction}

Developmental instability (DI) refers to small, random perturbations that arise during development [1]. The most widely used measure for DI is fluctuating asymmetry (FA). FA refers to small random deviations from perfect symmetry in bilateral symmetric traits (but also others such as radial symmetry [2]) and has received a lot of attention in many fields of Biology. The idea behind the use of FA is that the two sides of an organism represent two replicates of the same developmental process, and any deviation from symmetry is the outcome of random noise. A second-albeit less popular-measure of DI are the so-called phenodeviants, a term first coined by Lerner [3]. It refers to abnormal morphological deviations which initially were shown to increase with inbreeding [3]. Later, Rasmussen [4] concluded that the frequency of phenodeviants (further abbreviated as fPD) can serve as a measure of DI as well.

Because of its ability to reflect DI, FA has often been studied for its relationship with indicators of fitness and/or stress at both the population and individual level $[5,6]$. Various types of stress have been proposed to increase DI. These can be grouped into (1) genetic perturbations, such as mutations and inbreeding [7,8]; (2) adverse environmental conditions, such as pollutants, parasitism, and habitat disturbance [9-11]; and (3) maternal effects, including prenatal stress due to maternal exposure to teratogens, maternal infections, diseases and female age [12-14]. The relationship between FA and 
stress has been assessed in a large amount of studies, however yielding highly heterogeneous results. Indeed, the effects appear to be trait-, sex-, and importantly also stress-specific [5,6,11,15,16].

Especially, little is known about the consistency of the link between DI and maternal stress. While it has been concluded that poor maternal condition or maternal stress can impair developmental precision of the offspring, this conclusion was based on only a few studies, which were predominately conducted in humans. For example, it has been reported that maternal alcohol intake and smoking, advanced maternal age, maternal infectious and cardiovascular diseases, gestational diabetes and hypertension, and maternal obesity, correlate with increased FA levels in children [13,14,17-20]. A possible mechanism for the observed association between maternal stress and FA in children has been proposed and tested by studying DI in the offspring of mothers who experienced a natural disaster during pregnancy [20]. It was thought that prenatal stress may influence fetal development through the action of maternal glucocorticoids, which are steroid hormones that regulate metabolic, cardiovascular, homeostatic and immunological functions [21]. Contrary to expectations, maternal cortisol levels were negatively-instead of positively—related to the children's dermatoglyphic asymmetry (differences in fingertip ridge counts between left and right) [20]. However, maternal cortisol levels could only be determined several months after the natural disaster in a limited number of cases that fulfilled sampling criteria. More generally, because studies in humans are typically conducted retroactively, it is nearly always impossible to ensure the actual stress involved and the level at which it occurred. Therefore, conclusions solely based on these human studies can only be suggestive with respect to the underlying mechanism.

To tackle this question, experimental studies are needed which allow testing the causal relationship between (different levels of) maternal stress and DI. Unfortunately, such studies are scarce, and those that have been conducted mostly induce stress through a cortisol treatment. For example, FA increased in juveniles of Atlantic salmon after experimental maternal cortisol enhancement where the highest level of FA was observed in offspring that had been challenged with both high prenatal cortisol levels and hyperthermia [22]. Similarly, corticosterone or cortisol treatment, either through the mother or directly into the egg, has resulted in increased FA in hatchlings in chickens, Japanese quails and damselfish [23-25]. In a free-living species, high corticosterone levels were related to elevated FA levels of male nestlings in tree creepers [26]. These studies-albeit being very relevant as such-were not designed to study maternal stress as the cortisol/corticosterone was administered directly, without challenging the mothers in some way. More studies are clearly needed that experimentally evaluate the effect of different kinds of maternal stress on offspring DI. Also, the use of treatments inducing different levels of maternal stress is of interest to examine the dose-response curve of the (potential) DI-stress relationship.

Here, we investigate the relationship between maternal stress and both limb FA and fPD in rabbit fetuses from an experiment conducted within a reproductive toxicological framework. Pregnant does, which were assigned to three treatment groups (plus a control), received different concentrations of a drug under development. This experiment was chosen because the toxicological records showed that the administrated compound affected mainly maternal condition and did not induce major fetal malformations, which would suggest a direct toxic effect. Our aim was to assess the potential of measures of DI to reflect maternal stress. Specifically, we wanted to test (1) if there was an increase in fetal DI following maternal exposure to the toxic dose levels of a compound; (2) if there was a dose-response relationship; and (3) if there was a correlation between FA and FPD, our two measures of DI. In addition, we will discuss the potential value of DI as a "early warning system" in developmental toxicity studies where maternal condition/stress are a concern. The idea is that DI could serve as a predictor of changes in health in relation to stress that can be determined prior to more severe phenotypic outcomes [27] and, as such, may be used as a sensitive marker of developmental conditions [28]. 


\section{Materials and Methods}

\subsection{Study Species and Treatment}

Limb asymmetry was determined for fetuses of the New Zealand White rabbit (Oryctolagus cuniculus). These fetuses belonged to pregnant does that were exposed to a chemical compound in a toxicological experiment assessing its effects on embryo-fetal development. This experiment had been conducted within a reproductive toxicological framework at Janssens Pharmaceutica N.V. and the company allowed measuring FA of the limbs of their collection of fetuses. The compound had been under development for treatment of patients with diabetes mellitus type 2, but its development was stopped during an early clinical phase because of a lack of efficacy (further details cannot be disclosed due to company policy). The compound is no longer produced.

At the start of the experiment, pregnant does were randomly assigned to one of four treatments $(N=75$ does in total). The dose levels of the compound were 100, 500 and $1500 \mathrm{mg} / \mathrm{kg}$ in the low $(N=20)$, medium $(N=19)$ and high treatment $(N=16)$, respectively. The compound was formulated as a suspension in $0.5 \%(w / v)$ aqueous hydroxypropyl methylcellulose and administered once daily by gavage at a volume of $10 \mathrm{~mL} / \mathrm{kg}$ body weight. There was also a control group $(N=20)$, which received the vehicle according to the same dosing volume and treatment regime. Treatments were administrated orally on a daily basis during the period of organogenesis from day 6 until day 19 of pregnancy. Does were weighed daily and food consumption was monitored twice a week. On day 28 of pregnancy all does were sacrificed (such that all fetuses had the same age) and a necropsy was performed to examine litter data, including the number of corpora lutea, implants, early and late resorptions, and live and dead fetuses. All fetuses $(N=704)$ were weighed and examined for external, visceral and skeletal abnormalities. Evaluation of skeletal abnormalities and limb asymmetry was achieved by processing fetuses with the clearing and staining procedure during which fetuses are made transparent and bone tissue is stained with alizarin red $S$ [28,29]. The experiment has been approved by the ethical committee for animal experimentation of Janssen Pharmaceutica N.V. and was performed according to Good Laboratory Practices. Data from this study have also been reported elsewhere studying the genetic basis of DI and directional asymmetry [30,31].

\subsection{Limb Asymmetry}

To evaluate the FA of the cleared and stained fetuses, digital pictures of the limbs were taken in a standardized manner. To this extent, a digital camera (Nikon D300s camera with a Sigma 105 mm macro lens, Mapletree Anson, Singapore) was attached to a tripod at a fixed height and connected to a computer such that photographs could be made using the Nikon Camera Control Pro program (version 2.6.0) (Mapletree Anson, Singapore). An additional light source was placed underneath the fetus to ensure the visibility of the bone structure embedded in transparent tissue. Each fetus was positioned such that the fore- or hind limb was parallel to the camera lens (if necessary using small glass tubes for fixation). Repeated photographs were made for each specimen after independent repositioning of fore- and hind limbs. Using these digital images, the length of the left and right humerus, radius, ulna, femur, tibia and fibula was determined for each fetus in ImageJ version 1.46 (Bethesda, MD, USA) [32]. These limb bones were measured from the midpoint of the distal end until the midpoint of the proximal end (Figure 1). Measurements were taken by two operators (Jessica Bots or Matteo Breno) since a preliminary analysis showed no differences among operators (independent measurements were taken for 30 fetuses and correlation tests showed that results were highly comparable, all $p<0.001$ ). For a subset of individuals, all pictures were measured a second time to be able to determine measurement error $(\mathrm{N}=182)$, based on mixed regression models $[28,30,31,33]$. 


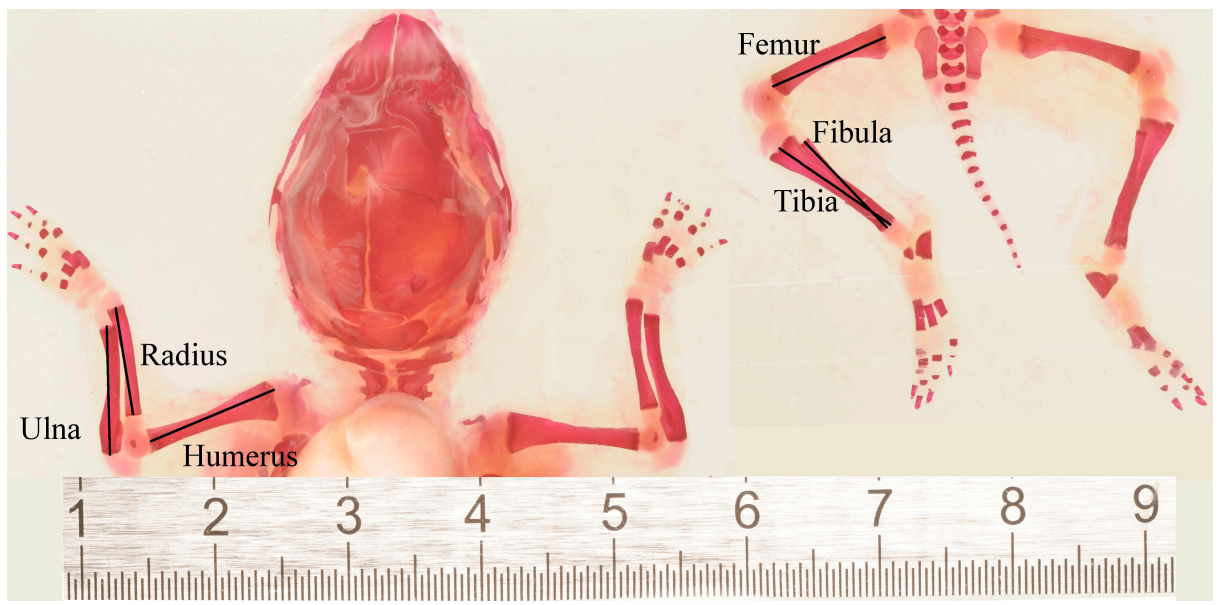

Figure 1. Illustration of a cleared and stained rabbit fetus with black bars indicating the traits measured on the fore limb (humerus, radius, ulna) and the hind limb (femur, tibia, fibula). The scale indicates the length in centimetres.

\subsection{Statistical Analysis}

All following analyses were conducted using unbiased individual asymmetry values (FAs), which were extracted from a mixed regression model [33]. Unbiased FA values are corrected for directional asymmetry (DA) and measurement error (ME), which could be determined at the level of individual measurements (based on repeated measurements of the same digital picture) and resulting from mounting and photographing the fetus (based on repeated pictures of the same individual). Significance of FA over ME and of the presence of DA was tested with a likelihood ratio test [33] (Table 1). Absolute values of the signed FAs were used as measures of DI for each trait studied. In addition, after standardisation, mean FA was calculated for fore- (i.e., using FA values of humerus, radius and ulna) and hind limbs (i.e., using FA values of femur, tibia and fibula), and across all six traits (i.e., using FA values of humerus, radius, ulna, femur, tibia and fibula).

Table 1. Overview of levels of measurement error (ME) as a result of positioning the fetus and taking a photograph $\left(\sigma^{2}\right.$ ME-photo. $)$ and measuring on a photograph $\left(\sigma^{2}\right.$ ME-meas. $)$ relative to real FA $\left(\sigma^{2} \mathrm{FA}\right)$, percentage of variation due to $\mathrm{ME}(\% \mathrm{ME})$, and levels of directional asymmetry (DA) in fore- and hind limb bones. Variance components $\left(\sigma^{2}\right)$ were multiplied by 1000 . Values highlighted in bold are statistically significant at $p<0.001$.

\begin{tabular}{cccccc}
\hline Trait & $\sigma^{\mathbf{2}}$ FA & $\sigma^{\mathbf{2}}$ ME-photo. & $\sigma^{\mathbf{2}}$ ME-meas. & \%ME & DA \\
\hline Humerus & $\mathbf{0 . 0 2 3 9}$ & 0.0105 & 0.0098 & 46 & $\mathbf{0 . 0 0 2 0}$ \\
Ulna & $\mathbf{0 . 0 6 4 8}$ & 0.0299 & 0.0134 & 40 & $-\mathbf{0 . 0 0 1 3}$ \\
Radius & $\mathbf{0 . 0 1 4 4}$ & 0.0037 & 0.0103 & 49 & $\mathbf{0 . 0 0 1 8}$ \\
Femur & $\mathbf{0 . 0 1 6 6}$ & 0.0019 & 0.0100 & 41 & -0.0001 \\
Tibia & $\mathbf{0 . 0 3 2 3}$ & 0.0136 & 0.0098 & 42 & $\mathbf{- 0 . 0 0 0 9}$ \\
Fibula & $\mathbf{0 . 0 1 5 8}$ & 0.0003 & 0.0209 & 57 & $\mathbf{- 0 . 0 0 0 8}$ \\
\hline
\end{tabular}

Firstly, we examined the effect of the different treatments on the condition of the pregnant does and the litters they produced. Specifically, it was tested whether there was a difference between treatments in food consumption (=total food consumed/food consumed before exposure) and weight gain (=original weight - final weight - uterus weight) of the does during pregnancy, pre-implantation and post-implantation loss, litter size and average pup weight per litter. All these analyses were conducted with a mixed Analysis of Variance model, except for average pup weight per litter where a mixed Analysis of Covariance model was used with litter size added as covariate. In each case, litter ID was added as random effect nested within treatment group, and comparisons based on F-tests. 
Furthermore, it was also examined whether any treatment led to an increase in the presence of minor and major abnormalities of the fetuses. Differences among treatments were analyzed with a mixed logistic regression model. In total, 20 types of major abnormalities and 88 types of minor abnormalities and variations were recorded, with the most common ones being 13 pairs of ribs, reduced ossification of metacarpal bones and rudimentary pubis. Major abnormalities occurred only in 11 fetuses $(1.5 \%)$ out of a total of 704 (one in the control, five in the low, two in the medium, and three in the high treatment), which had malformations such as muscular ventricular septum defect, scoliosis, fused ribs, absent spleen, or exencephaly. The frequency of the major abnormalities was thus very low and did not differ between treatments $(p=0.51)$. Therefore, they will not be analysed further. For minor abnormalities (which reflect phenodeviants) occurring in at least $5 \%$ of the cases, it was also tested whether there was any effect of treatment with a generalized linear mixed model. The latter observations include an unossified or rudimentary pubis, reduced ossification of metacarpal bones, a fused, rudimentary or absent sternum bone, unilateral or bilateral (rudimentary) 13th rib and small fetal size. Minor delays in ossification are not the result of missing bones and fall into the category of skeletal variations, which are often transient or repairable via postnatal skeletal remodelling [34], except for fused sternum bones.

Subsequently, potential increases in FA with treatment were examined using a series of linear mixed models. Analyses were first conducted for all traits separately, and were then repeated for the mean FA of the fore limb, the hind limb and across all six traits. In these models, unsigned FA was treated as a dependent variable, treatment as a fixed effect and litter as a random factor.

Finally, it was checked whether there was a relationship between FA and FPD. This was done using a linear mixed model approach, where the presence/absence phenodeviants (codified as 0 or 1 ) represented the fixed effect and litter the random factor. In addition, findings occurring in the same skeletal structure (e.g., rib or sternum bone) were grouped. Finally, separate analyses for the different categories of phenodeviants were performed.

All the models were run under the $R$ environment ( $R$ development Core Team, 2001 [35]) using the package lmerTest. Significance of fixed effect estimates were obtained by $F$-tests as well as by generating posterior distributions of each parameter using Markov Chain Monte Carlo (MCMC) methods (making use of the wrapping function available in the package language $\mathrm{R}$ [36]).

\section{Results}

Exposure to the compound predominately affected the general condition of the pregnant does. Maternal food consumption and weight gain significantly decreased with treatment, such that values were lower in the medium and high treatment compared to the control treatment. Also, average pup weight was significantly lower in the medium and the high treatment (Table 2). By contrast, no effect was observed on litter size, pre-implantation and post-implantation loss, thus indicating that the compound did not have any adverse effect on litter success (Table 2). There was a significant increase in $\mathrm{PPD}\left(\chi^{2}=10.756, p=0.01\right)$. Specifically, minor abnormalities and variations were more frequent in the medium $(p=0.013)$ and high treatment $(p=0.004)$ compared to the control treatment. No differences were found for the low treatment $(p=0.40)$. A detailed analysis of the fPD showed that this effect was driven by an increase in specific abnormalities. Indeed, treated fetuses more frequently showed reduced or delayed ossification of some bone structures, they more frequently had a 13th thoracic rib (bilaterally but not unilateral or rudimentary), and were smaller in size (Table 3). These observations are in agreement with results on fetal weight, because a reduction in fetal weight is related to incomplete ossification of the skeleton [37]. Variation in thoracic rib number also occurred at high frequency in the control treatment (i.e., a 13th thoracic rib, see Table 3), but this is not unusual since previous studies have shown a high rate of spontaneous variation in rabbits [38].

Only very limited evidence for an increase in limb FA with treatment could be found. Specifically, when analysing traits of the fore- and hindlimbs separately, only radius FA increased significantly with treatment (Table 4). Interestingly, FA was already higher in the low compared to the control treatment and did not further increase in the medium and high treatment. However, this result was no longer 
significant after Bonferroni correction. Furthermore, a similar pattern could not be found for any of the other studied traits, nor when grouping the traits within limbs (fore- or hind limb) or taking the mean FA across all traits (Table 4).

Table 2. Summary of the effect of the four treatments on the condition of the pregnant does and on the litters they produced. Values are presented as mean \pm standard deviation. Pre-implantation, post-implantation loss and weight gain are presented as relative values. Numerator and denominator degrees of freedom are 3 and 71 in all cases, except for average pup weight they are 3 and 70 resp. because litter size was added as a covariate. Values in bold are significantly different from the control treatment $(p<0.05)$.

\begin{tabular}{cccccc}
\hline Trait & Control & Low & Medium & High & $\boldsymbol{F}$ \\
\hline Maternal food consumption (g) & $5.65 \pm 0.87$ & $5.38 \pm 1.62$ & $\mathbf{4 . 7 5} \pm \mathbf{1 . 0 5}$ & $\mathbf{4 . 2 8} \pm \mathbf{1 . 6 9}$ & $\mathbf{3 . 9 6}$ \\
Maternal weight gain (g) & $-0.03 \pm 0.04$ & $-0.05 \pm 0.03$ & $\mathbf{- 0 . 0 7} \pm \mathbf{0 . 0 4}$ & $\mathbf{- 0 . 1 1} \pm \mathbf{0 . 0 6}$ & $\mathbf{1 0 . 4 4 5}$ \\
Litter size (n) & $9 \pm 2.55$ & $9.1 \pm 2.13$ & $9.05 \pm 1.51$ & $9.19 \pm 1.83$ & 0.0263 \\
Pre-implantation loss (\%) & $0.17 \pm 0.21$ & $0.16 \pm 0.16$ & $0.17 \pm 0.13$ & $0.09 \pm 0.1$ & 1.1372 \\
Post-implantation loss (\%) & $0.06 \pm 0.09$ & $0.08 \pm 0.1$ & $0.05 \pm 0.07$ & $0.11 \pm 0.11$ & 1.5164 \\
Average pup weight (g) & $31.4 \pm 2.8$ & $30.11 \pm 3.45$ & $\mathbf{2 7 . 7 3} \pm \mathbf{3 . 5 3}$ & $\mathbf{2 6 . 2 2} \pm \mathbf{4 . 4}$ & $\mathbf{1 1 . 2 7 5}$ \\
\hline
\end{tabular}

Table 3. Results of the detailed analysis of the effect of treatment on the presence of minor skeletal abnormalities occurring in at least $5 \%$ of the cases. Data are presented as a proportion of fetuses affected. Pearson's $\chi^{2}$ values in bold indicate that the presence of abnormalities differs between treatments $(p<0.05)$. Abbreviations refer to: pubis not ossified (pno), metacarpal bone reduced ossification (mro), 13th pair of ribs (13rib), one 13th rib (1_13rib), one rudimentary 13th rib (r_13rib), rudimentary pubis (rb), small sized (sz), sternum bone fused (sbf), sternum bone absent (sba), sternum bone rudimentary (sbr).

\begin{tabular}{ccccccccccc}
\hline Treatment & pno & mro & 13rib & 1_13rib & R_13rib & rb & sz & sbf & sba & Sbr \\
\hline Control & 0.02 & 0.12 & 0.56 & 0.13 & 0.09 & 0.13 & 0.01 & 0.03 & 0.07 & 0.11 \\
Low & 0.02 & 0.21 & 0.57 & 0.09 & 0.09 & 0.25 & 0.02 & 0.02 & 0.05 & 0.04 \\
Medium & 0.08 & 0.28 & 0.59 & 0.1 & 0.09 & 0.35 & 0.06 & 0.12 & 0.05 & 0.09 \\
High & 0.15 & 0.49 & 0.78 & 0.09 & 0.03 & 0.35 & 0.14 & 0.25 & 0.06 & 0.07 \\
$\chi^{2}{ }_{3}$ & $\mathbf{3 0 . 8 4}$ & $\mathbf{6 3 . 6 7}$ & $\mathbf{2 3 . 9 9}$ & 1.71 & 7.35 & $\mathbf{3 0 . 3 2}$ & $\mathbf{3 3 . 7 5}$ & $\mathbf{6 4 . 8 5}$ & 0.78 & 5.26 \\
\hline
\end{tabular}

Table 4. Overview of the effect of the four treatments on the FA of the six studied traits, the mean within the fore- and the hind limb, and the overall mean FA across all traits. Values in bold are significantly different from the control treatment $(p<0.05)$.

\begin{tabular}{cccccc}
\hline Trait & Control & Low & Medium & High & $\boldsymbol{\chi}^{\mathbf{2}}{ }_{\mathbf{3}}$ \\
\hline Humerus & 0.0032 & 0.0032 & 0.0031 & 0.0038 & 6.38 \\
Ulna & 0.0059 & 0.0054 & 0.0051 & 0.0056 & 1.86 \\
Radius & 0.0021 & $\mathbf{0 . 0 0 2 7}$ & $\mathbf{0 . 0 0 2 6}$ & $\mathbf{0 . 0 0 2 7}$ & $\mathbf{8 . 8 0}$ \\
Femur & 0.0028 & 0.0027 & 0.0031 & 0.0028 & 2.09 \\
Tibia & 0.0039 & 0.0036 & 0.0039 & 0.0036 & 0.61 \\
Fibula & 0.0026 & 0.0024 & 0.0027 & 0.0031 & 1.63 \\
Fore limb & 0.0038 & 0.0037 & 0.0035 & 0.0040 & 0.51 \\
Hind Limb & 0.0031 & 0.0029 & 0.0033 & 0.0032 & 1.63 \\
Overall & 0.0034 & 0.0033 & 0.0034 & 0.0036 & 2.46 \\
\hline
\end{tabular}

Even though a consistent relationship between FA and treatment was absent, it is still possible that FA is only increased in those fetuses showing phenodeviants. However, an overall analysis could not uncover such associations for FA within fore- or hind limbs, nor for the mean across all traits (Table 5). In addition, a similar analysis looking in detail at the different limb traits also failed to detect a consistent pattern (Table 5). Specifically, humerus FA was higher in fetuses with a rudimentary 
pubis, but did not significantly increase with any of the other variations. Similarly, femur FA was only augmented in fetuses with an unossified pubis and radius and tibia FA was only higher in fetuses with fused sternum bones. By contrast, ulna and fibula FA did not increase with fPD (Table 5).

Table 5. Overview of the relationship between the presence of phenodeviants and FA of the six studied traits as well as mean FA within the fore- and the hind limb and across all traits. Data are presented as the slope of the relationship. Values in bold are significantly different at $p<0.05$. Abbreviations refer to: overall presence of minor abnormalities (minor), pubis not ossified (pno), metacarpal bone reduced ossification (mro), 13th pair of ribs, one 13th rib, or one rudimentary 13th rib (13rib), rudimentary pubis $(\mathrm{rb})$, small sized (sz), sternum bone fused (sbf), sternum bone absent or rudimentary (sbar). Note that these categories differ slightly from previous analyses since abnormalities that were considered to have a similar origin were grouped together.

\begin{tabular}{ccccccccc}
\hline Trait & minor & pno & mro & 13rib & rb & sz & sbf & sbar \\
\hline Humerus & -0.0002 & -0.0003 & 0.0002 & 0.0005 & $-\mathbf{0 . 0 0 0 5}$ & -0.0007 & 0.0003 & -0.0003 \\
Ulna & 0.0002 & -0.0011 & 0.0000 & 0.0000 & 0.0001 & 0.0004 & -0.0007 & 0.0000 \\
Radius & 0.0003 & 0.0002 & 0.0004 & 0.0000 & 0.0000 & -0.0004 & $\mathbf{0 . 0 0 0 8}$ & 0.0001 \\
Femur & 0.0001 & $\mathbf{0 . 0 0 1 3}$ & 0.0003 & -0.0003 & 0.0001 & -0.0005 & 0.0000 & -0.0003 \\
Tibia & -0.0002 & -0.0005 & -0.0002 & -0.0006 & -0.0001 & 0.0004 & $\mathbf{0 . 0 0 1 0}$ & 0.0000 \\
Fibula & -0.0001 & -0.0002 & 0.0001 & 0.0001 & -0.0003 & 0.0003 & -0.0003 & 0.0001 \\
Fore limb & 0.0000 & -0.0004 & 0.0002 & 0.0002 & -0.0002 & -0.0004 & 0.0001 & 0.0000 \\
Hind & 0.0001 & 0.0002 & 0.0000 & -0.0002 & -0.0001 & 0.0002 & 0.0003 & -0.0001 \\
Limb & & & & & & & & \\
Overall & 0.0000 & -0.0001 & 0.0001 & 0.0000 & -0.0001 & -0.0001 & 0.0002 & -0.0001 \\
\hline
\end{tabular}

\section{Discussion}

In this study, we experimentally tested for the effects of reduced maternal condition on offspring limb fluctuating asymmetry and the frequency of phenodeviants. The application of fetal outcomes to study maternal toxicity was suggested over 30 years ago e.g., [39]. It is expected that compounds with a direct toxic effect to the foetuses increases mortality and major abnormalities [39]. Compounds that are toxic to the pregnant females only, are expected to reduce food consumption resulting in female weight loss. In this study, pregnant rabbits were exposed to toxic dose levels of a compound, which reduced food consumption causing weight loss, but neither increased fetal mortality nor resulted in more major abnormalities. Although we cannot exclude small toxic effects to the foetuses, this observation is in agreement with a compound causing maternal nutritional stress. Against a priori expectation, we could not detect a dose-related increase of offspring FA. In addition, the experiment increased the frequency of phenodeviants, with an elevated frequency of 13th rib anomalies, reduced ossification of several skeletal structures, lowered pup weight and size. Most of these abnormalities indicate growth retardation which is often transient. One exception was the premature fusion of sternal bones. Nevertheless, we failed to find a consistent association between fetal limb FA and these skeletal variations. These results therefore indicate that at least in this rabbit model and for the compound applied, maternal stress does not result in elevated DI levels of offspring.

This is an unexpected result because earlier studies that induced maternal stress through corticosterone or cortisol treatment in several bird and fish species, did find evidence for an increase in offspring DI [23-25]. In this study, the pregnant does responded to the treatment by feeding less and they gained less weight, which led to a reduction in pup weight and ossification. These observations are in agreement with studies in rabbits showing that reductions in maternal body weight can result in lowered fetal weight and transient alterations in ossification, representing a delay in development associated with growth retardation [37]. Also, food shortage generally increases baseline glucocorticoids $[40,41]$ and in mammals' cortisol passing the placental barrier is considered a primary origin of prenatal stress [41]. Hence, it can be hypothesized that a change in feeding regime or body weight gain may influence DI and result in higher FA levels and/or fPD through the action of glucocorticoids. Indeed, in Western Scrub-Jays (Aphelocoma californica) for instance, experimental 
food restriction during post-hatching development led to an increase in both baseline corticosterone levels and DI [42]. Similarly, the decreased maternal food consumption and weight gain observed in this experiment may have affected glucocorticoid levels and hence DI. Furthermore, it may also be possible that maternal stress disturbed developmental buffering of the fetuses in a manner unrelated to changes in glucocorticoid levels. Nevertheless, in contrast to these expectations, a consistent increase in offspring DI could not be detected in this study.

Several possible explanations for the absence of a relationship between maternal stress and offspring DI in this study can be put forward, yet appear unlikely. Firstly, an obvious difference with earlier studies is that we measured fetal DI. It has been proposed that fetal traits may more reliably reflect DI, because the effects of early stress may become obscured due to subsequent growth and mechanical loading [19]. Furthermore, a recent meta-analysis showed that studies focussing on FA-stress associations in humans (e.g., relationships with congenital abnormalities, maternal infections or hormone treatment) yielded the highest estimates of the relationship between FA and stress (i.e., highest effect sizes) among the studied categories [15]. Therefore, studying FA of fetal traits and associations with phenodeviants should have been a powerful tool to detect a relationship with maternal stress, if it would have existed.

Secondly, previous studies on the effects of maternal glucocorticoids have used birds and fish as a model system [22-25], which are oviparous animals. In contrast, here we studied maternal stress in a mammal. It has been shown in viviparous reptiles that live bearing provides a more stable environment for developing fetus, compared to related oviparous reptiles, which enhances viability and performance $[43,44]$. If parallels can be drawn towards developmental buffering in mammals, then the womb may be a very stable and controlled environment such that a uterine development may be more resilient to perturbations. Placental hormone function could play a critical role here, as it regulates the maternal-fetal energy balance [45]. In food-restricted pregnant females, the placental hormone function reduces the placental capacity to transport nutrients herewith retarding fetal growth [45] as observed here. In contrast to this possibility, a study of the macaque did find that daily mild stress, induced by capturing pregnant females, resulted in significantly higher dermatoglyphic asymmetry in the offspring [46]. Although insufficient data are currently available, the latter at least suggest that effects of experimentally induced maternal stress on offspring FA can emerge in mammals. Thus, different types of stress might have different effects on growth and developmental instability.

Thirdly, it is possible that, for an unknown reason, the New Zealand white rabbit is insensitive to stress in terms of increased FA. This is unlikely because in a similar experiment, exposing pregnant does of the New Zealand white rabbit to four treatments (control, low, medium, high) of a compound that caused major abnormalities in the fetuses but limited maternal problems, an effect of treatment on fetal limb FA could be found [28]. Interestingly, FA of the hind limbs was already higher in the low treatment than in the control, whereas abnormalities were induced in the high dose only [28]. These results are consistent with the "early warning system" hypothesis, which proposes that FA could serve as a predictor of changes in fitness and health $[10,27]$. However, the increase of FA was not proportional to the dose; indicating FA can reveal a stressor but provides little information on its magnitude [28]. Here, radius FA followed a similar pattern in that it was already augmented in the low compared to the control treatment, but did not further increase in the medium and high treatment. Nevertheless, this result could not be confirmed in an analysis combining all studied traits or solely forelimb traits and it was no longer significant after Bonferonni correction, suggesting that FA plays little role as an "early warning system" when studying maternally mediated fetal outcomes. It is possible that only the alteration of some developmental pathways, but not others, is able to affect DI. Evidence for this possibility was found in a study on deceased human fetuses [19], where limb FA was augmented for only four out of the 17 studied categories of developmental abnormalities. Alternatively, in terms of models of developmental instability, the so-called morphogenetic drift model for skeletal asymmetry can be in agreement with a lack of a relationship between FA and stress as early in development as we studied rabbit fetal FA. Under this model, it is expected that 
developmental imprecision accumulates during the continued growth such that effects on foetuses will become expressed later in life [47]. Interestingly, skull shape FA in mice under nutritional stress did not increase in magnitude, but the spatial pattern of FA was altered [48]. Unfortunately, this aspect could not be studied here because we used linear FA measures. Future research should (also) focus on spatial/shape aspects of FA (see also [48]).

\section{Conclusions}

The failure to reject the null hypothesis in this study most likely reflects the absence of a moderate to strong effect of maternal nutritional stress on DI in this study, since all requirements (large sample size, experimentally induced stress, and repeated measures of multiple traits) to detect an association with high statistical power were fulfilled. While both measures of DI (FA and frequency of phenodeviants) did not consistently reflect maternal stress, and were not inter-correlated, one phenodeviants did show a slight signal. The premature fusion of sternum bones increased significantly with dose and was correlated with FA in two traits (but not to average FA). The study of specific (and probably very rare phenodeviants) thus appear to warrant further study in larger experiments. Nevertheless, in spite of a very strong effect on maternal condition, the treatment had no or very limited effect on DI, and any effect is likely to be trait specific. We argue that this null result might be due to the fact that we have studied a viviparous mammal, where in utero development remains highly buffered in spite of a deteriorated maternal condition where the placenta might play an important role in regulating the maternal-fetal energy balance. Further research is clearly required to support this claim.

Acknowledgments: This work benefited from a research grant from the University of Antwerp (BOF-KP 4382). MB holds a Ph.D. Fellowship from the Research Foundation-Flanders (FWO). J.B. received a postdoctoral fellow with the Research Foundation-Flanders (FWO). Luc De Bruyn provided valuable advice on taking photographs of the rabbit fetuses. We are indebted to Peter Delille for the technical help.

Author Contributions: J.B., M.B., L.D.S. and S.V.D. conceived and designed the experiment; L.D.S. performed the experiments; J.B. and M.B. performed all measurements and analyzed the data; L.D.S. contributed materials; J.B., M.B. and S.V.D. wrote the paper.

Conflicts of Interest: The authors declare no conflict of interest.

\section{References}

1. Polak, M. Developmental Instability: Causes and Consequences; Oxford University Press: Oxford, UK, 2003.

2. Savriama, Y.; Klingenberg, C.P. Beyond bilateral symmetry: Geometric morphometrics for any type of symmetry. BMC Evol. Biol. 2011, 11, 280. [CrossRef] [PubMed]

3. Lerner, I.M. Genetic Homeostasis; Edinburgh and London: Oliver \& Boyd: New York, NY, USA, 1954.

4. Rasmussen, M. Frequency of morphological deviants as a criterion of developmental stability. Hereditas 1960, 46, 511-535. [CrossRef]

5. Lens, L.; van Dongen, S.; Kark, S.; Matthysen, E. Fluctuating asymmetry as an indicator of fitness: Can we bridge the gap between studies? Biol. Rev. 2002, 77, 27-38. [CrossRef] [PubMed]

6. Van Dongen, S. Fluctuating asymmetry and developmental instability in evolutionary biology: Past, present, future. J. Evol. Biol. 2006, 19, 1727-1743. [CrossRef] [PubMed]

7. Leamy, L.J.; Klingenberg, C.P. The genetics and evolution of fluctuating asymmetry. Annu. Rev. Ecol. Evol. Syst. 2005, 36, 1-21. [CrossRef]

8. Pertoldi, C.; Kristensen, T.N.; Andersen, D.H.; Loeschcke, V. Developmental instability as an estimator of genetic stress. Heredity 2006, 96, 122-127. [CrossRef] [PubMed]

9. Møller, A.P. Parasitism and developmental instability of hosts: A review. Oikos 1999, 77, 189-196. [CrossRef]

10. Lens, L.; van Dongen, S.; Matthysen, E. Fluctuating asymmetry as an early warning system in the critically endangered Taita Thrush. Conserv. Biol. 2002, 16, 479-487. [CrossRef]

11. Hoffman, A.A.; Woods, R.E. Associating environ- mental stress with developmental stability: Problems and patterns. In Developmental Instability: Causes and Consequences; Polak, M., Ed.; Oxford University Press: Oxford, UK, 2003; pp. 387-401. 
12. Parson, P.A. Maternal age and developmental variability. J. Exp. Biol. 1962, 39, 251-260.

13. Livshits, G.; Davidi, L.; Kobyliansky, E.; Ben-Amital, D.; Levi, Y.; Merlob, P. Decreased developmental stability as assessed by fluctuating asymmetry of morphometric traits in preterm infants. Am. J. Med. Genet. 1988, 29, 793-805. [CrossRef] [PubMed]

14. Singh, D.; Rosen, V.C. Effects of maternal body morphology, morning sickness, gestational diabetes and hypertension on fluctuating asymmetry in young woman. Evol. Hum. Behav. 2001, 22, 373-384. [CrossRef]

15. Van Dongen, S.; Gangestad, S.W. Human fluctuating asymmetry in relation to health and quality: A meta-analysis. Evol. Hum. Behav. 2011, 32, 380-398. [CrossRef]

16. Thornhill, R.; Møller, A.P. Developmental stability, disease and medicine. Biol. Rev. 1997, 72, 497-548. [CrossRef] [PubMed]

17. Wilber, E.; Newell-Morris, L.; Streissguth, A.P. Dermatoglyphic asymmetry in fetal alcohol syndrome. Biol. Neonate 1993, 64, 1-6. [CrossRef] [PubMed]

18. Kieser, J.A.; Groeneveld, H.T.; Da Silva, P.C. Dental asymmetry, maternal obesity and smoking. Am. J. Phys. Anthropol. 1997, 102, 133-139. [CrossRef]

19. Van Dongen, S.; Wijnaendts, L.C.D.; Ten Broek, C.M.A.; Galis, F. Fluctuating asymmetry does not consistently reflect severe developmental disorders in human foetuses. Evolution 2009, 63, 1832-1844. [CrossRef] [PubMed]

20. King, S.; Mancini-Marïe, A.; Brunet, A.; Walker, E.; Meaney, M.J.; Laplante, D.P. Prenatal maternal stress from a natural disaster predicts dermatoglyphic asymmetry in humans. Dev. Psychopathol. 2009, 21, 343-353. [CrossRef] [PubMed]

21. Benderlioglu, Z. Fluctuating asymmetry and steroid hormones: A review. Symmetry 2010, 2, 541-553. [CrossRef]

22. Eriksen, M.S.; Espmark, Å.M.; Poppe, T.; Braastad, B.O.; Salte, R.; Bakken, M. Fluctuating asymmetry in farmed Atlantic salmon (Salmo salar) juveniles: Also a maternal matter? Environ. Biol. Fishes 2006, 81, 87-99. [CrossRef]

23. Eriksen, M.S.; Haug, A.; Torjesen, P.A.; Bakken, M. Prenatal exposure to corticosterone impairs embryonic development and increases fluctuating asymmetry in chickens (Gallus gallus domesticus). Br. Poult. Sci. 2003, 44, 690-697. [CrossRef] [PubMed]

24. Satterlee, D.G.; Hester, A.; Leray, K.; Schmidt, J.B. Influences of maternal corticosterone and selection for contrasting adrenocortical responsiveness in Japanese quail on developmental instability of female progeny. Poult. Sci. 2008, 87, 1504-1509. [CrossRef] [PubMed]

25. Gagliano, M.; McCormick, M.I. Hormonally mediated maternal effects shape offspring survival potential in stressful environments. Oecologia 2009, 160, 657-665. [CrossRef] [PubMed]

26. Helle, S.; Suorsa, P.; Huhta, E.; Hakkarainen, H. Fluctuating feather asymmetry in relation to corticosterone levels is seks-dependent in Eurasian treecreeper (Certhia familiaris) nestlings. Biol. Lett. 2010, 6, 521-524. [CrossRef] [PubMed]

27. Clarke, G.M. Relationships between developmental stability and fitness-Application for conservation biology. Conserv. Biol. 1995, 9, 18-24. [CrossRef]

28. Breno, M.; Bots, J.; de Schaepdrijver, L.; van Dongen, S. Fluctuating asymmetry as risk marker for stress and structural defects in a toxicological experiment. Birth Defects Res. B Dev. Reprod. Toxicol. 2013, 98, 310-317. [CrossRef] [PubMed]

29. Dawson, A.B. A note on the staining of skeleton of cleared specimens with alizarin red S. Stain Technol. 1926, 1, 123-124. [CrossRef]

30. Breno, M.; Bots, J.; van Dongen, S. Between-family variation and quantitative genetics of developmental instability of long bones in rabbit fetuses. Biol. J. Linn. Soc. 2013, 109, 33-42. [CrossRef]

31. Breno, M.; Bots, J.; van Dongen, S. Heritabilities of directional asymmetry in the fore- and hindlimbs of rabbit fetuses. PLoS ONE 2013, 8, e76358. [CrossRef] [PubMed]

32. Abramoff, M.D.; Magalhaes, P.J.; Ram, S.J. Image Processing with ImageJ. Biophotonics Int. 2004, 11, 36-42.

33. Van Dongen, S.; Molenberghs, G.; Matthysen, E. The statistical analysis of fluctuating asymmetry: REML estimation of a mixed regression model. J. Evol. Biol. 1999, 12, 94-102. [CrossRef]

34. Carney, E.W.; Kimmel, C.A. Interpretation of skeletal variations for human risk assessment: Delayed ossification and wavy ribs. Birth Defect Res. B Dev. Reprod. Toxicol. 2007, 80, 473-496. [CrossRef] [PubMed] 
35. Baayen, R.H.; Davidson, D.J.; Bates, D.M. Mixed-effects modelling with crossed random effects for subjects and items. J. Mem. Lang. 2008, 59, 390-412. [CrossRef]

36. The $\mathrm{R}$ project for statistical computing. Available online: http://www-r-project.org (accessed on 17 August 2016).

37. Cappon, G.D.; Fleeman, T.L.; Chapin, R.E.; Hurt, M.E. Effects of feed restriction during organogenesis on embryo-fetal development in rabbit. Birth Defect Res. B Dev. Reprod. Toxicol. 2005, 74, 424-430. [CrossRef] [PubMed]

38. Palmer, A. Spontaneous malformations of the New Zealand White rabbit: The background to safety evaluation tests. Lab. Anim. 1968, 2, 195-206. [CrossRef]

39. Khera, K.S. Maternal toxicity in humans and animals: Effects on fetal development and criteria for detection. Teratig. Carcinog. Mutagen. 1987, 7, 287-295. [CrossRef]

40. Wingfield, J.C.; Maney, D.L.; Breuner, C.W.; Jacobs, J.D.; Lynn, S.; Ramenofsky, M.; Richardson, R.D. Ecological bases of hormone-behavior interactions: The 'emergency life history stage'. Am. Zool. 1998, 38, 191-206. [CrossRef]

41. Painter, R.C.; Roseboom, T.J.; de Rooij, S.R. Long-term effects of prenatal stress and glucocorticoid exposure. Birth Defects Res. C Embryo Today 2012, 96, 315-324. [CrossRef] [PubMed]

42. Pravosudov, V.V.; Kitaysky, A.S. Effects of nutritional restrictions during post-hatching development on adrenocortical function in western scrub-jays (Aphelocoma californica). Gen. Comp. Endocrinol. 2006, 145, 25-31. [CrossRef] [PubMed]

43. Shine, R. A New Hypothesis for the evolution of viviparity in reptiles. Am. Nat. 1995, 145, 809-823. [CrossRef]

44. Shine, R. Does viviparity evolve in cold climate reptiles because pregnant females maintain stable (not high) body temperatures? Evolution 2004, 58, 1809-1808. [CrossRef] [PubMed]

45. Dimasuay, K.G.; Boeuf, P.; Powell, T.L.; Jansson, T. Placental responses to changes in the maternal environment determine fetal growth. Front. Physiol. 2016, 7, 1-9. [CrossRef] [PubMed]

46. Newell-Morris, L.L.; Fahrenbruch, C.E.; Sackett, G.P. Prenatal psychological stress, dermatoglyphic asymmetry and pregnancy outcome in the pigtailed macaque (Macaca nemestrina). Biol. Neonate 1989, 56, 61-75. [CrossRef] [PubMed]

47. Hallgrímsson, B. Fluctuating asymmetry in the mammalian skeleton-Evolutionary and develop-mental implications. Evol. Biol. 1998, 30, 187-251.

48. Gonzalez, P.N.; Lotto, F.P.; Hallgrímsson, B. Canalization and developmental instability of the fetal skull in a mouse model of maternal nutritional stress. Am. J Phys. Anthropol. 2014, 154, 544-553. [CrossRef] [PubMed]

(C) 2016 by the authors; licensee MDPI, Basel, Switzerland. This article is an open access article distributed under the terms and conditions of the Creative Commons Attribution (CC-BY) license (http://creativecommons.org/licenses/by/4.0/). 